\title{
Lo singular del empleo de los cirujanos dentistas
}

The unique employment of dental surgeons

A singularidade do uso de cirurgiões dentários

DOI: $\underline{\text { http://dx.doi.org/10.23913/rics.v6i11.50 }}$

Cándido González Pérez ${ }^{1}$

Universidad de Guadalajara, México

candido195913@yahoo.com.mx

\section{Resumen}

Este trabajo es producto de una investigación que en su primera parte se basó en una metodología cuantitativa al aplicar una encuesta a la totalidad de estudiantes de primer ingreso de la carrera de cirujano dentista, en la segunda en una cualitativa donde se entrevistó a los egresados de dos generaciones y a un empleador que ofreció información desde su perspectiva como investigador, docente, funcionario y patrón. Se contrastan los resultados con dos teorías antagónicas entre sí donde una privilegia el actuar del individuo para basar sus sustentos, y la otra le otorga el papel determinante a la sociedad donde el seno familiar es definitorio para que los estudiantes accedan al éxito escolar y laboral. Una de las características de este escrito es que la totalidad de la información presentada fue extraída de primera mano.

Palabras clave: formación universitaria, empleo, capital cultural.

\section{Abstract}

This work is the product of an investigation that in its first part was made using a quantitative methodology when applying a survey to the totality of Dental Surgeon Major freshmen. In the second part, there's a qualitative analysis of the graduates of two generations and an employer who offered information from his perspective as a researcher,

\footnotetext{
${ }^{1}$ Investigador del Centro Universitario de Los Altos de la Universidad de Guadalajara, miembro del Sistema Nacional de Investigadores.
} 
faculty member and employer. The results are contrasted with two opposing theories where one privileges the individual's action to base his or her livelihoods, and the other gives the determining role to the society where the family sine is defining for students to access school and work success. One of the characteristics of this writing is that all of the information presented was obtained first-hand.

Key words: University education, employment, cultural capital.

\section{Resumo}

Este trabalho é o resultado de uma investigação que primeira parte foi baseada em uma metodologia quantitativa, aplicando uma pesquisa para todos cirurgião-dentista carreira calouros, o segundo de uma qualitativa onde os graduados foram entrevistados em duas gerações e um empregador que forneceu informações de sua perspectiva como pesquisador, professor, diretor e padrão. os resultados com duas teorias conflitantes juntos são contrastadas onde privilégios ato do indivíduo a basear seus meios de subsistência, ea outra dá o papel de uma sociedade onde a família está definindo para os alunos a acessar a escola e trabalhar sucesso. Uma característica deste trabalho é que toda a informação apresentada foi extraído em primeira mão.

Palavras-chave: ensino universitário, de emprego, de capital cultural.

Fecha recepción: Agosto 2016

Fecha aceptación: Enero 2017 


\section{Introducción}

\section{Las expectativas}

Para describir las expectativas que se forman los estudiantes de la Carrera de Cirujano Dentista, me baso en una encuesta que fue aplicada a la totalidad de los alumnos de primer ingreso en el Centro Universitario de Los Altos ${ }^{2}$ durante el mes de agosto del año 2016. La información más importante para explicar lo que buscan debe obtenerse de dos fuentes: sus características individuales y lo que piensan de su formación universitaria. De la primera, llama la atención que una proporción elevada de los estudiantes (43\%) proviene de poblaciones que no pertenecen al área de influencia del Centro Universitario de Los Altos; esta cualidad es común observarla en las carreras del área de la salud y es que debido a las altas evaluaciones académicas que se les requiere para el ingreso, los puestos son competidos entre un mayor número de alumnos y provienen de localidades más distantes. Por ejemplo, de las carreras de administración y psicología únicamente el 10\% proviene de municipios que no corresponden a Los Altos Sur, y de ingeniería agroindustrial el 5\%, mientras que de medicina $47.7 \%$. Otra característica que distingue inclusive al interior del área de la salud, es que por ejemplo los de medicina optaron por esa carrera como primera opción el $96 \%$ mientras que los de cirujano dentista solamente el $76 \%$, y del $24 \%$ que no lo hizo, habían optado en su mayoría precisamente por la de medicina.

Con respecto a la manera de pensar de los estudiantes, quienes optaron por la formación de dentistas eligieron su formación en primer lugar porque les gusta ayudar a otras personas, porque les llamaba la atención, por la atracción que ejerce el área de la salud, por el mercado laboral y por tradición familiar como se puede observar en la gráfica. Contrasta en gran medida la forma de pensar de estudiantes que aspiran culminar otro tipo de formación como lo es la contaduría pública que le dan todo el peso a la orientación vocacional y sus necesidades particulares; la de negocios internacionales por el mercado laboral y motivos propios, o la ingeniería agroindustrial donde sus inclinaciones individuales fueron definitorias.

\footnotetext{
${ }^{2}$ El abanico de pregrado corresponde a: abogado, administración, cirujano dentista, contaduría pública, enfermería, ingeniería agroindustrial, ingeniería en computación, ingeniería en sistemas pecuarios, veterinaria, medicina, negocios internacionales, nutrición y psicología.
} 
Figura 1. Motivos para elegir la profesión.

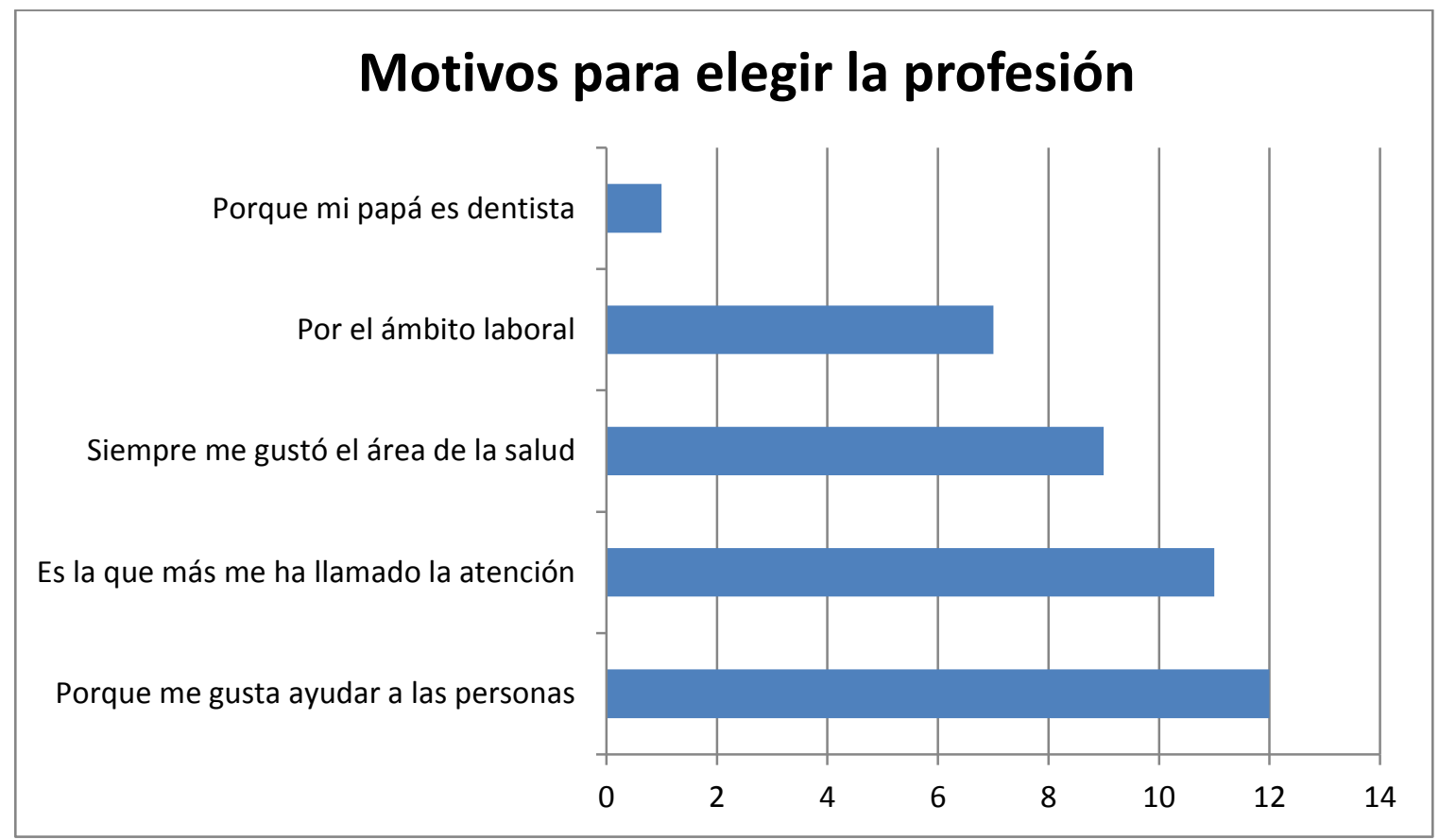

Fuente: información obtenida en forma directa a través de la encuesta aplicada en agosto del 2016.

La situación principal por la que eligieron el Centro Universitario de Los Altos y que por desgracia logra un consenso entre la mayoría de los estudiantes de primer ingreso, es porque está ubicado con cercanía a su población de origen. Las carreras que fueron elegidas por esta circunstancia y por lo menos por la mitad de los alumnos son: contaduría pública, enfermaría, ingeniería agroindustrial, ingeniería en computación, negocios internacionales, nutrición y psicología. 
Figura 2. ¿Por qué se eligió el CUALTOS?

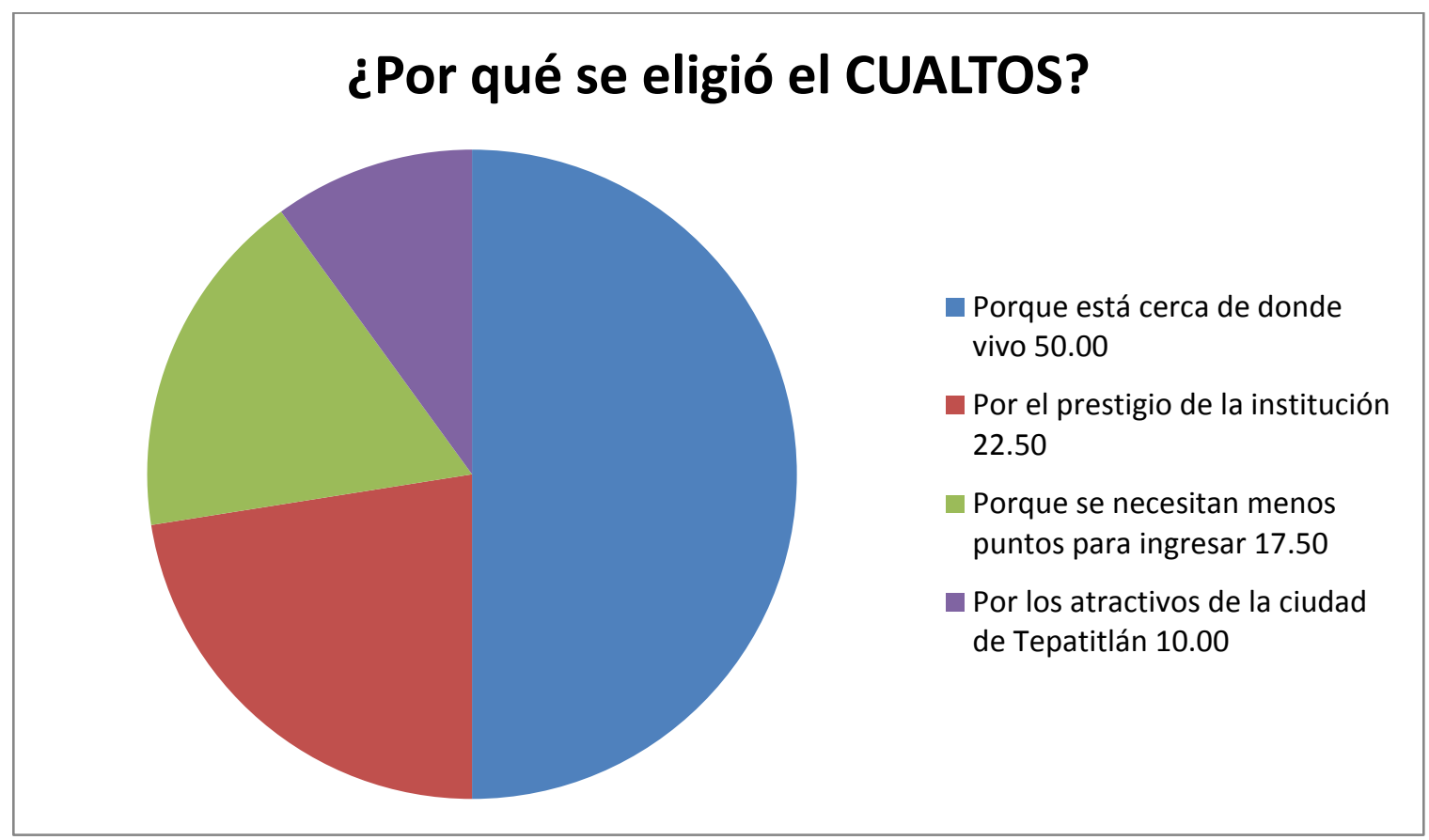

Fuente: información obtenida en forma directa a través de la encuesta aplicada en agosto del 2016.

Lo que esperan aprender de la profesión es recibir una formación de alta calidad (37.50\%), ayudar a los demás (20\%), los aspectos laborales (20\%), los prácticos (17.50\%) y conocer las patologías de las personas (5\%). Estas cualidades están a tono con los motivos que manifestaron para haber elegido esta carrera. Estas cifras conforman el preámbulo de las expectativas que se han formado para cuando culminen su carrera y que en términos particulares, se concentran en dos opciones: creando su consultorio privado o trabajando para el sector público ${ }^{3}$. Esta información es relevante porque sirve de comparación para los resultados obtenidos de entrevistas con los estudiantes ya egresados de dos generaciones.

\footnotetext{
${ }^{3}$ Con respecto a una pregunta particular en la encuesta a estudiantes de primer ingreso donde se les pide su opinión con respecto a las cosas que le desagradan de su formación, las tendencias principales demuestran precisamente su inquietud por lo que les depara el mercado laboral y por las carencias económicas que perciben van a tener durante su tránsito por la universidad.
} 
Figura 3. ¿Cómo se ven los estudiantes al culminar sus estudios?

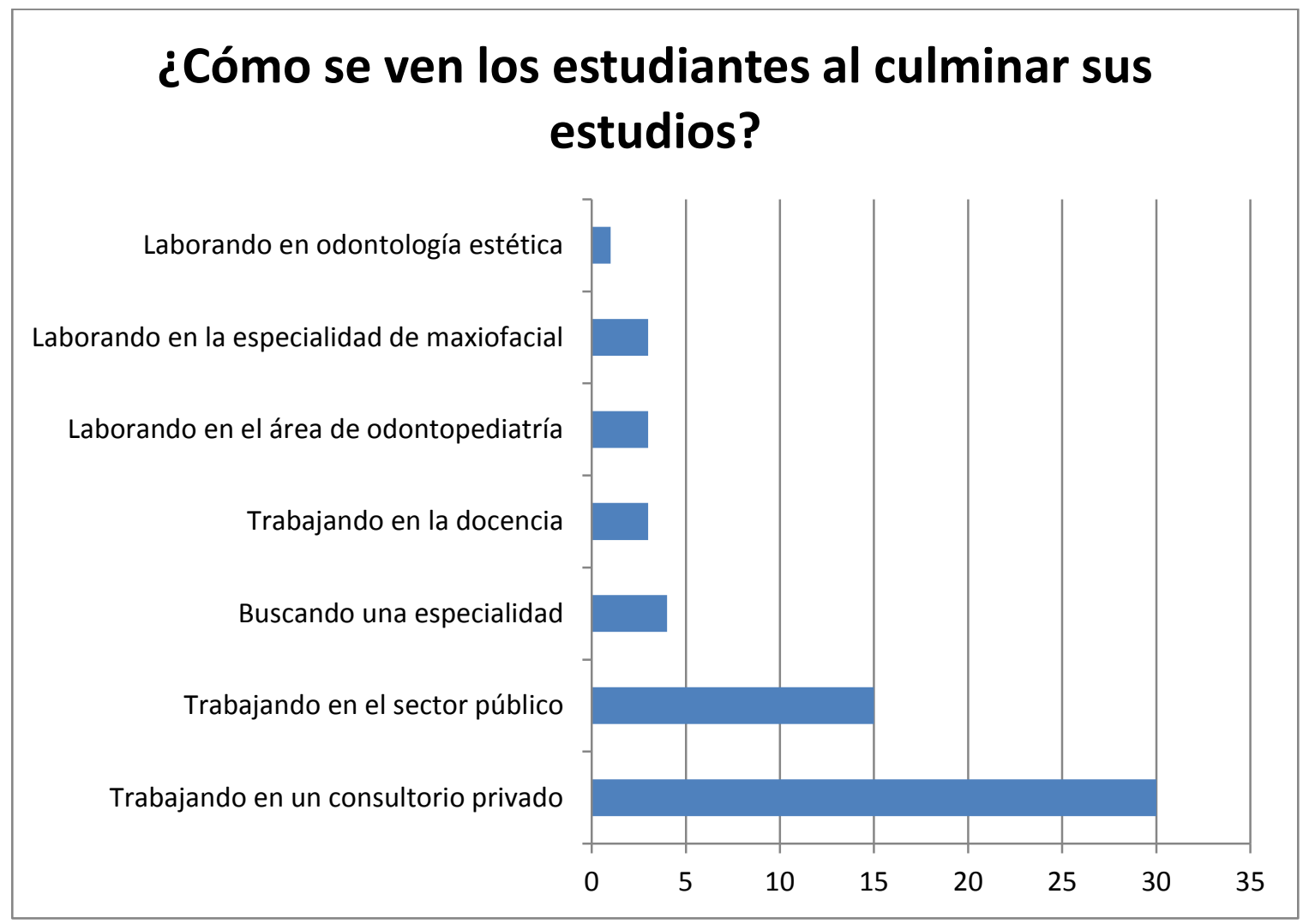

Fuente: información obtenida en forma directa a través de la encuesta aplicada en agosto del 2016.

\section{Características del empleo de los cirujanos dentistas}

En este apartado se presentan los resultados de la aplicación de entrevistas a egresados de la carrera de cirujano dentista del Centro Universitario de Los Altos correspondientes a dos generaciones: la del año 2012 y la de $2013^{4}$. Son muy jóvenes y eso influye en su incorporación al empleo, el promedio de edad es de 26 años para la primera y de 23.17 la segunda. Renglones arriba se mencionó que el $43 \%$ de los alumnos de primer ingreso provenían de localidades externas a la región Altos Sur y ellos significa que la proporción ha ido disminuyendo puesto que la proporción de quienes egresaron en 2012 y 2013 fue del $56.25 \%$. De cualquier manera, son proporciones elevadas al compararse con la

\footnotetext{
${ }^{4}$ De un total de 92 egresados fue posible localizar a 49 y ellos conforman nuestro universo de estudio en los resultados aquí presentados. Los entrevistados representan el 53.25\% del total.
} 
conformación de los grupos ajenos al área de la salud. Un cambio sustancial en el que se debe poner atención en el área de la orientación vocacional es que el $92.5 \%$ de los egresados optó por la opción universitaria de la odontología como su primera elección, mientras que de los estudiantes de primer ingreso, solamente el 76\% lo hizo, esto significa que cada vez son más quienes buscan incorporarse en un tipo de formación y luego modifican su elección.

Los aspectos que tienen mayor influencia en la incorporación al mercado laboral son los siguientes: los recursos económicos para el mantenimiento de los estudios provino de las siguientes fuentes, por parte de los padres el $60 \%$, por una combinación de proveerse emolumentos ellos mismos y sus padres el $16 \%$, por medio de becas estudiantiles más el concurso de los padres el 14\%, y por medio de ellos mismos el 10\%. En otros estudios de seguimiento a egresados que se han llevado a cabo en este mismo centro universitario, la proporción de los estudiantes que son apoyados exclusivamente por sus padres es sensiblemente menor, por ejemplo, los de la carrera de contaduría pública son el $22 \%$ (González y Ortega; 2015).

Conocer la escolaridad de los padres de familia es fundamental para confrontar los conocimientos teóricos de dos corrientes del pensamiento que tienen influencia en la relación que existe entre formación universitaria y obtención del empleo, son las del Capital Humano y del Capital Cultural, sus representantes Schultz y Bourdieu (1997, 2002) ofrecen ópticas alternativas que deben ponerse a debate. En particular, el espectro que ofrece la descripción de grados académicos tanto del padre como de la madre, sirve para resaltar dos aspectos, primero, el bajo nivel en promedio, y segundo, que la mujer cuenta con la proporción más baja. No obstante ello, es de hacer notar que al hacer una comparación de esta característica en lo particular con la observada en los egresados de otras carreras, es sensiblemente más alta. Por ejemplo, los padres de los egresados de contaduría pública ninguno cuenta con nivel superior de educación y la concentración mayor se ubica en estudios de primaria (González y Ortega; 2015); de enfermería y administración aunque sí cuentan algunos padres con licenciatura, la mayor concentración se ubica también en estudios de primaria (Machaen y González; 2016. González y Padila, 2016). 
Figura 4. Escolaridad de los padres.

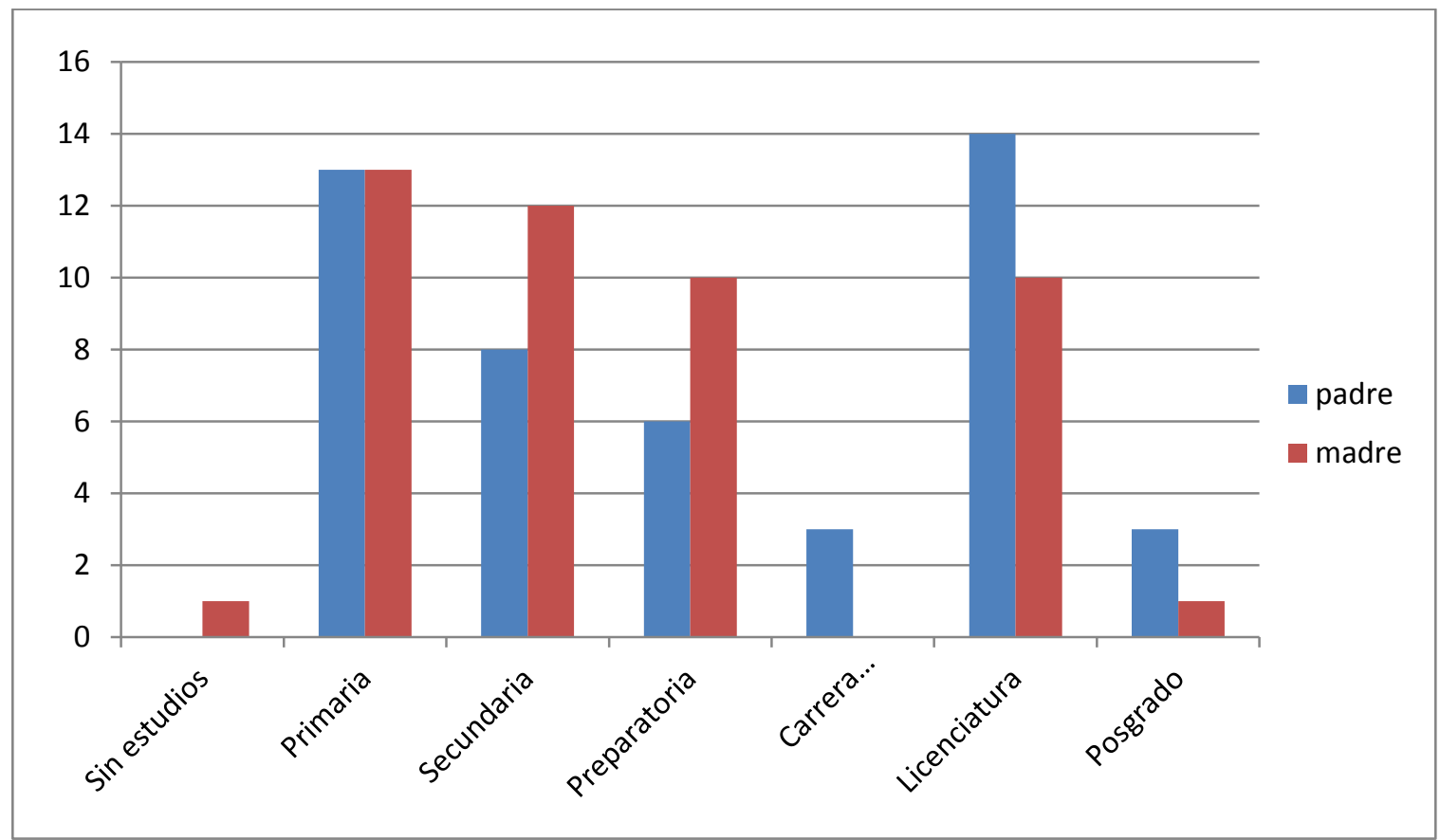

Fuente: información obtenida en forma directa a través de entrevistas directas.

La escolaridad de los padres y el ámbito laboral en el que se desarrollan son los dos puntos más importantes para determinar el origen social de los estudiantes. Los egresados de odontología de manera muy similar a los de ingeniería en computación, cuentan con los estándares escolares menos bajos de los padres de los egresados del Centro Universitario de Los Altos (González y Carrillo; 2015). Para la corriente de pensamiento denominada del Capital Cultural, ambas características (la escolaridad y el espacio de trabajo de los padres) son determinantes para proveer a los hijos las herramientas necesarias que los dirijan a obtener éxito en su formación y un buen futuro en su posición social, principalmente la adquisición de un buen empleo (Bourdieu, 1997). Los espacios laborales en los que se desarrollan los padres o tutores de los egresados de odontología están caracterizados porque una proporción baja la ocupan como trabajadores calificados, en particular: odontólogos, profesores o profesionistas ejerciendo en forma liberal (24.44\%); el resto son empleados sin calificación entre los que destacan los ganaderos, quienes se desempeñan en los oficios tales como carpinteros, torneros, electricistas, choferes, y los comerciantes. 
Figura 5. Espacios laborales de los padres o tutores.

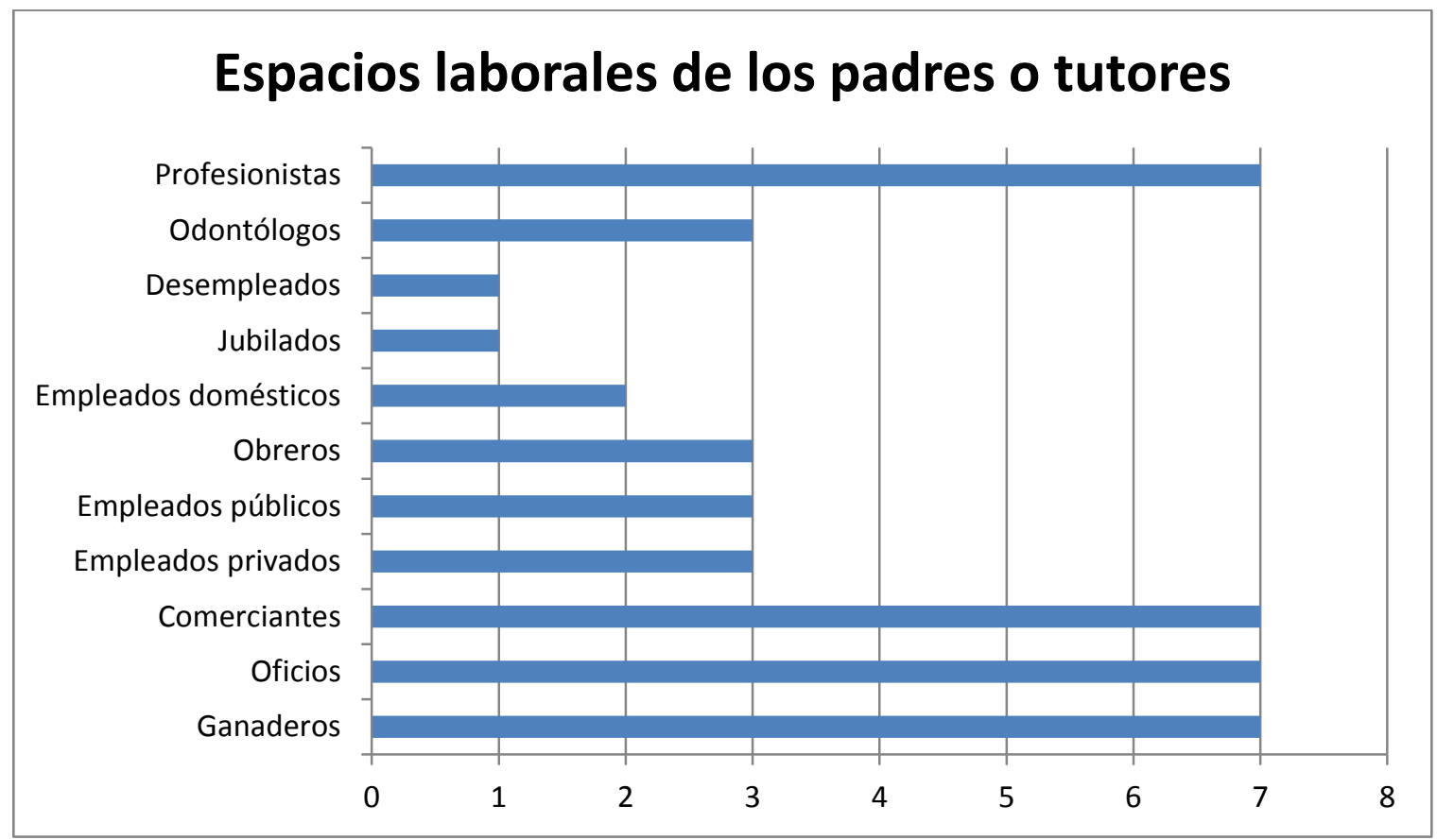

Fuente: información obtenida en forma directa a través de entrevistas directas.

A continuación se detalla la manera en que se distribuye el empleo de los odontólogos egresados. El principal objetivo de realizar estudios a egresados es el conocer el grado de influencia que tuvo la formación universitaria en el empleo; para el caso de las dos generaciones que fueron entrevistadas para la realización de este trabajo destacan tres aspectos, el primero, que una proporción considerablemente alta está desempleado (el $46.80 \%$ ), el segundo, quienes trabajan en relación directa con su formación representan un $29.79 \%$, y tercero, que representan la proporción más baja de los profesionistas empleados que han egresado del Centro Universitario de $\operatorname{Los}$ Altos $^{5}$ y de las que se han elaborado estudios de seguimiento a egresados. Para terminar con esta parte del empleo profesional de los odontólogos, no se puede dejar pasar un aspecto de importancia y que está relacionado con lo que se denomina currículum oculto. Este concepto significa que hay saberes que adquieren los profesionales de las diferentes áreas y que no están contemplados en los diferentes programas de estudio, es decir, que son transmitidos por los docentes a través de

\footnotetext{
${ }^{5}$ Entre las carreras que se consideran son: contaduría pública, veterinaria, negocios internacionales, ingeniería en computación, administración, psicología y nutrición.
} 
experiencias personales y se enseñan con la práctica diaria, de los que coloquialmente se les llama tips pero que no se les debe analizar de forma superficial. Los conocimientos originados por la vía descrita se aplican constantemente y son la representación viva de la práctica profesional; si el interés principal de los estudios de seguimiento a egresados es conocer el grado de influencia del conocimiento adquirido en la universidad y su aplicación en el medio, esto significa que estamos hablando de los aspectos más importantes. En concreto, los saberes que los estudiantes afirman haber obtenido sin que hayan sido materia de estudio formal en el desarrollo de su carrera y que aquí describo, se exponen asumiendo que los primeros son los que más se mencionaron, es decir, en forma descendente: aprendieron en prácticas particulares cómo contener hemorragias, cómo tratar a los niños como pacientes, cómo reducir la ansiedad, un maxilofacial los llevaba a su consultorio a enseñarlos con ejemplos prácticos, casos prácticos de endodoncia, casos de prostodoncia, casos teóricos que eran mostrados en forma práctica, cómo desgastar dientes en zonas particulares, y finalmente, un profesor los ponía a trabajar como auxiliares para que fueran conociendo cada uno de los pasos en los diferentes problemas que se presentaban.

Figura 6. Empleo de los egresados.

\section{Empleo de los egresados}

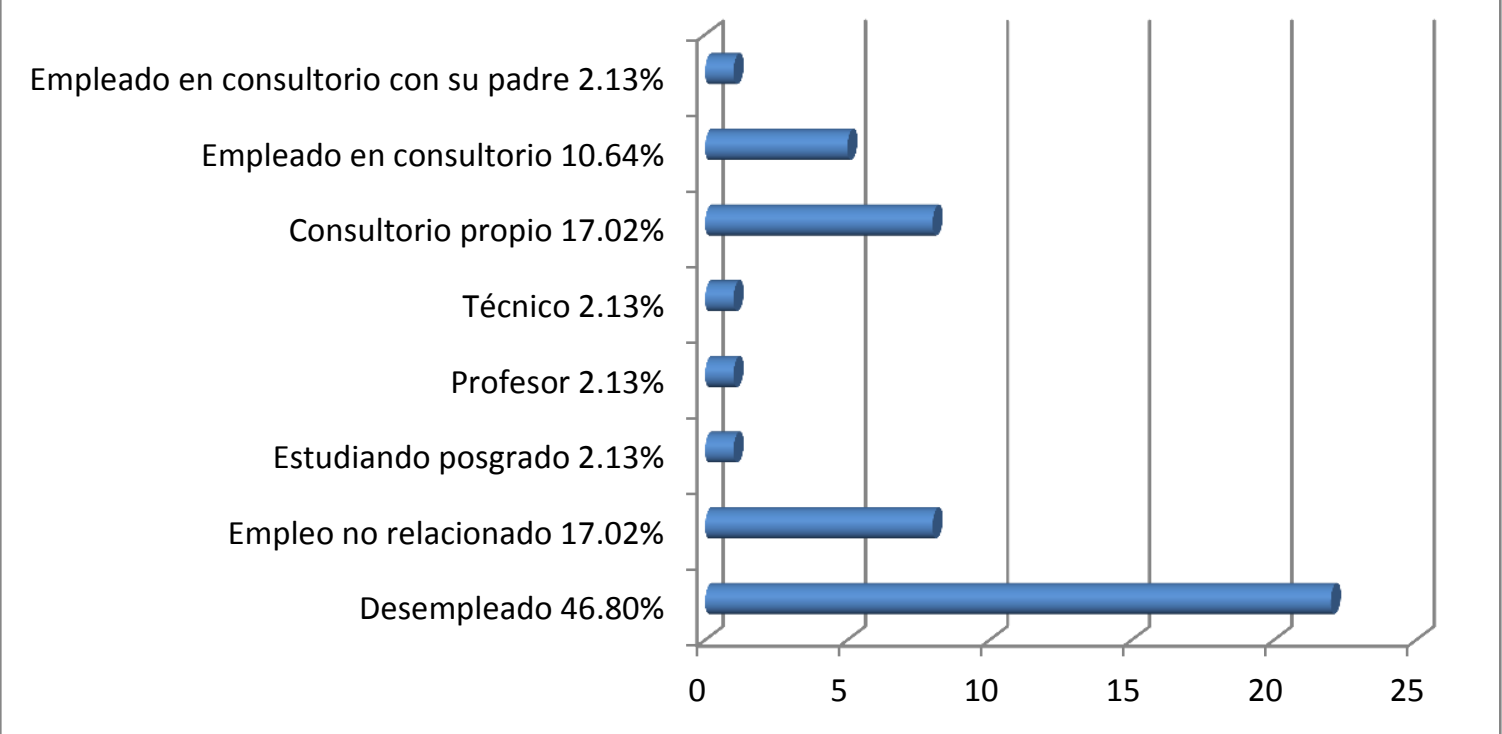

Fuente: información obtenida en forma directa a través de entrevistas directas. 


\section{Experiencias laborales}

En este apartado se expone información referida a la experiencia de un profesional que ha conjuntado etapas de lo más variadas en la odontología: como profesor, investigador, empleado, propietario de una clínica, empleador y funcionario. En el inciso anterior había mencionado que uno de los principales objetivos de un estudio de seguimiento a egresados es conocer el grado de conocimientos adquiridos por los profesionistas, ahora presento el caso de que el otro lado de la moneda no es menos importante: el de los empleadores. ¿Qué conocimientos se adquirieron y se han utilizado? La respuesta o respuestas a esta interrogante la puede dar un empleador. ¿Cuáles son los avatares por los que debe pasar un profesionista de la odontología? Los conoce un profesional que se ha desempeñado durante un largo período. ¿Qué se debe hacer para continuar con la preparación? Las tres preguntas son contestadas por la misma persona porque engloba las diferentes facetas de un profesional de la cirugía dental y además explica de forma particular cuáles son los principales obstáculos que atraviesan los egresados para incorporarse al mercado de trabajo.

La carrera de odontólogo, por ser un área clínica es muy diferente con los empleadores, para ellos sino es en lo particular, solamente la Secretaría de Salud, el Seguro Social y el ISSSTE $^{6}$ son los que pueden darles trabajo. En las instituciones privadas es un poco más complicado porque ahí va de por medio el prestigio de una persona o un grupo. Por ejemplo en mi caso yo tengo un dentista que está de tiempo completo conmigo, yo le doy empleo. No hay muchas maneras diferentes de hacerlo, en mi caso, yo soy el dueño de todo, de las instalaciones, de todo el equipo y yo le pago un sueldo. En otros casos, si se asocian, tienen que comprar entre varios el equipo, dividirse lo de la renta y todos los gastos. Pero entran en igualdad de circunstancias en todo sentido y obligaciones, claro también en beneficios. En mi caso no, él trabaja para mí, yo soy empleador.

Hay dos factores bien importantes a tener en cuenta en eso del empleo, primero, los jóvenes al egresar, para empezar un negocio en grande, tienen que equipar un consultorio, eso es muy difícil. Ellos enfrentan esa realidad. Es muy caro arrancar un negocio aun cuando sea desde lo más básico y que consiste en un sillón dental, un

\footnotetext{
${ }^{6}$ Instituto de Seguridad y Servicios Sociales de los Trabajadores del Estado.
} 
aparato de rayos $\mathrm{X}$ (incluso uno usado), un autoclave, es decir lo mínimo indispensable; esto significa más de 100,000 pesos más lo que les cueste por los gastos forzados de renta, mantenimiento, promoción y todo eso. La segunda parte son los clientes, los egresados se desesperan porque piensan: tuve solamente un paciente en una semana. Dicen, ya tengo muchos gastos y con lo poco que entra no puedo sostenerme. Entonces, de todo lo que se gasta ahora hay que ver el otro lado: las ganancias. Y cuando se empieza eso va muy despacio. Ellos no entienden esa parte, porque siempre empieza uno con la familia, atendiendo a los primos, atendiendo a los amigos, los hermanos, cobrando más barato. De esa manera es como empiezan, forzosamente. Muchos se desesperan y desertan. No ven los beneficios pronto y deciden claudicar. Más cuando salen y ya traen un compromiso. Piensan en ganar dinero en un ritmo más rápido, piensan que como ya invirtieron en la carrera, ahora es a recuperar y se enfrenta a uno con la realidad. Esos dos factores son bien importantes, es la principal razón para que deserten.

Generalmente los jóvenes que estudian odontología son quienes tienen una capacidad un poco mayor que la del promedio porque es una carrera cara, requiere de muchos materiales que hay que invertir, el equipo es caro, no es nada económico, entonces los muchachos se enfrentan a una realidad un poco difícil. La realidad en la parte económica es uno de los obstáculos que enfrentan los alumnos. A mí me ha tocado firmar bajas voluntarias ni siquiera en la parte del egreso sino antes, al principio o a la mitad de la carrera. Es triste ver que van y dicen:

-Ya no pude, me quiero dar de baja.

Entonces yo les digo: haz un esfuerzo mayor y me dicen:

-No, ya no puedo.

Desde primero, quinto, sexto semestre, truenan en lo económico y van para fuera. La familia y la gente que los apoya ya no encuentran la forma de solventarlos y es una de las razones por las que he firmado bajas. La cantidad de deserciones es alta. Con nosotros se requiere mucha infraestructura, en las carreras de administración o de abogado no se necesita relativamente nada, pero con nosotros, no les podemos 
proveer de instrumental, eso lo deben adquirir ellos y ahí es donde está lo difícil, en lo económico se les complica.

La universidad no tiene la capacidad económica de decir yo te presto esto o yo te presto el instrumental, yo te pongo el equipo. Nosotros en el área clínica tenemos lo básicos pero no podemos entregarles el equipo. En laboratorio o en las clínicas tenemos lo mínimo. Ellos necesitan comprar material, articuladores, muchas cosas en el transcurso, esas cosas son caras. En un semestre deben invertir entre 5,000 y 10,000 pesos, que no es mucho pero que hay familias a quienes les significa eso el comer o no comer. No es mucho para algunos, pero hay familias que sí. Los papás dicen con 10,000 mil pesos comemos a lo mejor dos meses. Yo he firmado bajas definitivas siendo la única razón la económica.

Los muchachos a veces vienen mal orientados, tal vez lo que escucharon no fue lo correcto y a veces piensan otra cosa, pero cuando se enfrentan con la realidad ya ven que era diferente la cosa. Pueden pensar al escuchar los comentarios que en odontología las cosas van bien, sí, pero conocen los casos exitosos, ya cuando ven que la mayoría tiene que invertir desde la carrera para tomar impulso, ya no es como lo habían escuchado. Lo que quieren y más de alguno ha desertado es porque no han sabido bien lo que quieren, a veces no es hasta el momento de salir sino que es desde que están en la carrera. Hay muchos filtros que ellos desconocen y cuando se topan con ellos, la cosa cambia mucho. El factor económico es topar con pared porque es desde que son estudiantes y también al momento del egreso, si no tienen el recurso para armar un consultorio, es lo mismo que les pasó desde que eran estudiantes que no tenían el dinero para comprar los materiales y el equipo. Y luego un consultorio rentado. Por ejemplo, al paso del tiempo yo soy dueño de mi consultorio, de mi equipo; lo he ido armando poco a poco y por fortuna, me ha ido bien. No sucede lo mismo con todos. Topan y se desesperan. El avance tecnológico es gigante, pero por lógica, cada avance significa equipo más caro, sí te sirve mucho más, pero vale mucho más, es una carrera para muchos inalcanzable.

Sobre lo de currículum oculto, en odontología se ve mucho. Eso sucede en la parte clínica, que de repente sabe uno un tip, una estrategia, un truco o un método, se le 
puede llamar de diferentes maneras de resolver una problemática directamente en una clínica con un paciente. Es una manera de resolver un problema en el trabajo y que nunca lo puedes conocer a través de la teoría. Es algo de muy día a día en odontología. Finalmente eso ya va de una manera aterrizado en la parte clínica. De hecho yo tuve una buena relación con mis profesores, algunos de ellos me invitaban a ver cómo eran sus prácticas en sus consultorios, a nivel de licenciatura y a nivel de especialidad yo iba a trabajar con algunos de mis profesores a cuatro manos con sus pacientes y en su clínica particular, eso es una experiencia muy difícil de igualar, es que ahí trabajas de la mano con tu profesor y lo haces aprendiendo muchísimo. Es muy diferente que te lo expliquen a que lo veas y lo hagas tú mismo junto a tu mentor. Lo hacíamos en sus clínicas particulares. Lo que yo aprendí ahí, es muy difícil que lo llegues a conocer dentro del salón de clases. Eso que asimilé con ellos, es imposible que lo entiendas en el aula, no hay como la práctica. Lo que aprendí ahí me sigue ayudando en la experiencia que adquirí, y es cierto, esas cosas no las aprende uno en los libros, la práctica en mucho más rica en varios aspectos. Y puedo decir que eso mismo se puede aplicar a nivel de especialidad, no solo en el curso de la carrera.

Para ingresar a la Carrera de Cirujano Dentista se requiere un puntaje de los más altos en la universidad, siempre el más elevado está entre medicina, contaduría, nutrición y odontología. De entre la gran cantidad de oferta profesional, la de odontología es de las más difíciles de ingreso. Desgraciadamente a lo de los requisitos de puntaje en la admisión, hay que agregarle lo de los ingresos económicos. Hace dos meses firmé otra baja de un muchacho que me dijo que ya había picado por aquí y por allá y que definitivamente sus familiares y quienes lo apoyaban ya no pudieron sostenerlo. Y son bajas definitivas, se alejan por completo, no es que reprueben sino que se les hace imposible mantenerse por los recursos que se necesitan. ¿Ya buscaste alternativas? Les pregunto.

-Ya, he platicado con mis padres, mis allegados que me pueden apoyar y es definitivo, tengo que abandonar la carrera- me dicen. 
Yo egresé en el año 1995, soy de Tepatitlán, egresé de la carrera en lo que es ahora el Centro Universitario de Ciencias de la Salud que antes no existía, era solamente la Universidad de Guadalajara, yo egresé y como a los 8 años se abrió esta posibilidad en Tepatitlán, aquí es nueva la opción. Tengo especialidad en Endodoncia también en Guadalajara en la Universidad de Guadalajara. Hay como 6-8, bueno, hay muchas más, de las que yo conozco en nuestra institución son alrededor de 6 u 8. Aquí en CUALTOS tenemos dos. En cuanto a especialidades, estamos a buen nivel en la Universidad de Guadalajara, inclusive aquí en el Centro Universitario de Los Altos, las dos que tenemos están reconocidas en el Padrón de Excelencia del Consejo Nacional de Ciencia y Tecnología, son de muy buen nivel, fueron evaluadas muy seriamente y logramos la incorporación. Son los únicos dos programas de nuestro centro universitario que han alcanzado ese nivel. Nosotros hemos ido completando el equipo con gente de aquí, empezamos con algunos de fuera, de nuestra misma institución pero no de nuestro mismo centro, y al paso del tiempo hemos ido ya completando un buen equipo de casa. Los requisitos son muy altos, y nosotros logramos ser admitidos. Hicimos equipo con gente de Guadalajara y siguen viniendo pero al paso del tiempo, ya tenemos una generación que egresó y entonces ahora hemos preparado a nuevos profesores de tiempo completo y le estamos dando forma de tal manera que cada vez requerimos menos de gente de fuera. Son los únicos dos programas de calidad, son los de odontopediatría y endodoncia. De hecho los implementamos desde cuando empecé yo como coordinador de la carrera, eso fue hace como tres años; le propuse a la rectora anterior a la doctora Mara, creyó en mí, me apoyaron y sí lo pudimos lograr. Lo hicimos a contra de viento y marea, hubo gente que se oponía. Ahora ya se ve que sí lo pudimos lograr, pero hay que decirlo, hubo gente que estuvo en contra. Hemos ido equipando lo más que podemos porque es una de las cosas que revisan siempre cuando se solicita la incorporación de un programa en el padrón de excelencia. Hemos gestionado aquí y allá y vamos avanzando bien, estamos comprometidos con nuestros programas porque nosotros los hicimos. Ya es más fácil cuando se va consolidando, cuando va empezando te creen menos. Ha habido gestiones externas y sí ha habido apoyo de mucha gente, no obstante los que estuvieron en contra. Como programa de reciente creación sí le 
entramos, ahora sigue mantenernos y luego la consolidación. Ante CONACYT somos el orgullo, no fue fácil lograr lo que coronamos. Dentro de lo difícil, ya lo gramos lo fácil que era entrar, ahora hay que llegar a la consolidación. Ya hemos tenido premios a nivel nacional e internacional con nuestros alumnos, inclusive en el propio Centro Universitario de Ciencias de la Salud, nos trajimos primero y segundo lugar concursando con los estudiantes de la especialidad, fuimos y llevamos trabajos y nos trajimos un primero y un segundo lugar a nivel de posgrados, a nivel de pasantes nos trajimos el primer lugar y a nivel de licenciatura un segundo lugar, todos estudiantes de aquí de CUALTOS. Los jueces fueron de allá y finalmente nos trajimos los primeros lugares. A nivel nacional en lo que es la asociación mexicana de endodoncia nos trajimos un primer lugar en el congreso de este año. A nivel internacional, concursamos en Barcelona el año pasado y nos trajimos un tercer lugar. Se publica en el baner de CUALTOS y en los spots de aquí de los medios locales. Yo puedo afirmar que somos el ejemplo a seguir. Hay que mantenernos y a seguir adelante.

Yo empecé aquí en Tepatitlán a trabajar desde cuando estaba dando mi servicio social. Había una oficinita en la casa de mis papás, ahí empecé. Inicié un diciembre cuando estaba como pasante, terminaba mi pasantía en julio y de ahí, a los cinco meses ya tenía mi consultorio. Arranqué atendiendo pacientes como pasante. En abril del año que le siguió, renté un consultorio en la Clínica de Los Altos aquí en Tepatitlán, con un apoyo total del doctor Arturo Bayardo y la señora Reyna su esposa, me apoyó en todo absolutamente y le renté un local, empecé a hacer relaciones, a ganarme la confianza de la gente y duré casi diez años ahí y después tuve la oportunidad de comprar la propiedad junto con mis hermanos a un lado exactamente de la casa de mis papás, compramos la propiedad, construimos empezando de ceros, todo, desde abajo, ya diseñado para lo que es, no adaptado, un arquitecto nos construyó. Ya van a ser 14 años ahora en abril y ahí seguimos, yo ya en mi clínica propia, con equipamiento propio y ahora incluso con la posibilidad de contratar personas. Ahora yo estoy trabajando y contrato personas que atiendan mi clínica. Yo estoy trabajando en la universidad y mi consultorio bien equipado está dando atención a clientes porque ahí tengo personas trabajando. Cuando estaba en la clínica del doctor Bayardo era yo el único odontólogo, había otros profesionales pero 
eran de otras áreas de la medicina, como dentista solamente yo. Todo lo demás era del equipo médico, había médicos de varias especialidades. Nosotros rentábamos y ahí aparecía en el directorio de la clínica que se prestaban esos servicios, así como yo había varios que ofrecían sus conocimientos. El único compromiso era que cuando se requería atención mayor ahí mismo se les ofrecía el servicio completo, es decir, ya la hospitalización. Es una práctica muy buena porque uno está en una clínica que ofrece una gran cantidad de servicios donde cada quien es responsable de lo suyo pero a la administración le servía también porque ahí mismo se daba la atención completa. Es de eso que se dice "ganar-ganar". Mi compromiso era pagar mi renta, todo el equipamiento era mío, la contabilidad era mía, la administración era propia, cada quien hace lo suyo. De los casi 10 años que estuve ahí, un médico internista estaba ya ahí, sigue en ese espacio, sigue rentando su consultorio, él a sus pacientes los ingresa ahí en el mismo hospital. Ahí los sigue atendiendo pero su compromiso es atender ahí mismo. Estoy seguro que al $98 \%$ de sus pacientes ahí los atiende. A la muerte del doctor Bayardo su sobrino Mario Caboada los sigue atendiendo, ahora ya construyó su propia clínica a media cuadra y sigue dando el servicio.

Yo soy cirujano dentista por la influencia familiar, mi padre es médico, mi abuelito era médico familiar; mi padre sigue activo. Tengo dos hermanos médicos ya con especialidad, tenía un tío que era dentista, murió hace doce años, era hermano de mi papá. Todos trabajamos aquí en Tepatitlán, hemos ejercido siempre aquí. Desde mi bisabuelo, fue de los primeros médicos que ha habido aquí en Tepatitlán, él tuvo la primera botica que había, inclusive mi padre aún conserva los libros donde se asentaban los registros de los medicamentos que se preparaban ahí. Los libros con las fórmulas originales, con su puño y letra. Te preparaban medicamento lo mismo para una persona que para una vaca, era para la atención veterinaria también. Se hacían las fórmulas y mi padre tiene los libros donde mi bisabuelo conservaba la información formal, la oficial y también la de sus fórmulas propias. Ya al paso del tiempo empezaron a aparecer las farmacias y las boticas se fueron cerrando. A mi abuelo le empezó a interesar más la parte clínica, ya no lo de farmacia y la botica la quitaron. Mi abuelito como médico se dedicó a la medicina y nunca se enfocó a lo de farmacia. Mi abuelito ejerció toda su vida profesional aquí en Tepatitlán hasta que murió. 
Entonces los Martín del Campo somos del área de la salud desde mi bisabuelo, mi abuelo, mi padre, mis hermanos y algunos tíos. Yo desde niño me gustaba jugar que con los carritos y que llevaba los enfermos y los atendía, siempre me gustó, desde niño, claro, por la influencia familiar. De hecho a mí me gustaba más el área de medicina porque yo veía a mi papá y claro, era mi ídolo, era mi ejemplo a seguir, entonces yo quería estudiar medicina, pero luego mi hermano mayor que entra a medicina y el que sigue también y que se mete el primero a la especialidad y ya trabajaba, y entonces el segundo ya iba a empezar también su especialidad y fue donde yo pensé como que médico ya era mucho. La odontología yo la tenía como una segunda alternativa. Finalmente yo veía que a ellos los llamaban por alguna situación de emergencia y aunque estuviéramos en una fiesta o en la Navidad o incluso ya dormidos en la casa y tenía que ir a atender y yo pensé, voy a ser profesional pero en el día voy a trabajar y en la noche a dormir. Decidí odontología pero por la influencia y por el gusto que adquirí en la familia.

\section{Confrontación de la Teoría del Capital Cultural}

Para comprobar si la Teoría del Capital Cultural ofrece luces sobre nuestro objeto de estudio, describo en estas líneas dos tipos de confrontación, la primera analizando por separado el grupo de estudiantes que presentó el mayor éxito escolar en el desarrollo de su formación profesional, y la segunda observando por separado el grupo de estudiantes que proviene de una familia en la cual sus padres o por lo menos alguno de ellos tuvo preparación universitaria. Se hace la presentación en la forma antes descrita debido a que Pierre Bourdieu el padre de la teoría citada, afirma que los estudiantes que provienen de un medio cultural elevado son los que tienen mayores posibilidades de tener éxito tanto escolar como laboral. Basados en estas afirmaciones, sus seguidores afirman que la universidad se ha conformado como una institución que reproduce las desigualdades sociales (Casillas, et.al; 2007).

Del primer grupo antes descrito, los que tuvieron el mayor éxito escolar, corresponde a 6 egresados quienes obtuvieron las evaluaciones más altas de toda su generación. Si las predicciones de Bourdieu fueran afirmativas, la mayoría de ellos deberían provenir de un seno familiar en el cual sus padres habrían obtenido un grado escolar elevado y se 
desempeñarían en un ámbito laboral por encima del promedio. La primera de ellas es hija de padre y madre que estudiaron solamente la primaria y su padre se dedica a la ganadería en pequeño, es decir, no proviene de un medio familiar favorable; en cuanto a su desempeño profesional, actualmente se encuentra desempleada. El segundo de los casos corresponde a un joven de quien su padre estudio primaria y su madre es analfabeta; el oficio del padre es herrero y el egresado no cuenta con trabajo. La tercera proviene de una familia en la cual sus padres estudiaron hasta la preparatoria, su padre se dedica a la construcción como albañil y ella cuenta con un trabajo en el cual ofrece consultas los fines de semana. El cuarto es hijo de una señora que estudió licenciatura y su padre cuenta con posgrado; el oficio de su padre es odontólogo y el egresado trabaja apoyándolo en su consultorio. La quinta proviene de una señora que estudió secundaria y su padre solamente educación primaria; el oficio del padre es empleado en un pequeño comercio de venta de artículos de plástico; la joven instaló su propio consultorio en sociedad con otros amigos y se desempeña en su ámbito laboral totalmente de acuerdo a su formación. La escolaridad del último de ellos es primaria para su madre y secundaria para su padre, mismo que se desempeña como mecánico; ella se dedica de lleno a la odontología y su especialidad es en atención a pacientes adultos. En resumen, solamente un caso de los seis descritos es congruente con los designios de la Teoría del Capital Cultural, el resto queda total o parcialmente fuera de esa lógica, en resumen, no hay concordancia entre las afirmaciones de la corriente teórica y la realidad observada en este caso particular. Casillas (2007) cataloga a los alumnos que a pesar de provenir de un medio social difícil porque sus padres no tuvieron acceso a la universidad y su empleo es de los más bajos en la escala general, como "Héroes" o "Pobres Exitosos". La Teoría del Capital Humano por su parte, ofrece explicaciones más adecuadas en cuanto que deja todo el peso de sus postulados en decir que las personas cuando eligen estudiar, están dejando de obtener recursos económicos porque dejan de trabajar; al renunciar a un salario, están apostando por el futuro porque al egresar siendo ya trabajadores calificados deben obtener remuneraciones mayores y con eso se compensa su elección de haber abandonado el empleo años antes. Formichela (2013) afirma que los empresarios pagan dos tipos de salarios: uno para quienes cuentan con estudios básicos, y otro más alto para quienes accedieron a los universitarios. Para esta corriente del pensamiento el individuo es el más importante como objeto de análisis, pone 
en el centro de la discusión el hecho de que son las personas cada una, sin influencia familiar, quienes deciden su futuro y quienes acceden al éxito escolar y/o laboral, siempre lo hacen por méritos propios. ${ }^{7}$ Por el contrario los postulados de Bourdieu señalan que la ventaja que tienen los individuos se basa en la acumulación de capital cultural en el hogar de las personas ${ }^{8}$.

El segundo grupo de egresados está constituido también por 6 personas, la característica que los separa del anterior es que en este caso los estudiantes no tuvieron éxito escolar, están por debajo del promedio. La cualidad que le da contenido a este nuevo equipo es que son hijos de personas que tuvieron acceso a la educación superior. El primero de ellos y que cumple con esta segunda categoría, es especial porque es el único que cumple también con haber tenido éxito escolar, es el que ya habíamos descrito que su padre tiene posgrado, su madre licenciatura, que trabaja en el consultorio de su padre y al mismo tiempo estudia un posgrado. El segundo es hijo de padre y madre que estudiaron licenciatura y no está trabajando porque se encuentra cursando un posgrado. Del tercero, su padre estudió hasta la preparatoria y su madre cursó una carrera técnica, está trabajando en su propio consultorio después de haberse preparado durante año y medio como auxiliar en uno privado. La cuarta es hija de un odontólogo, trabajó como maestra de inglés simultáneamente que apoyaba en el consultorio de su padre, ya se independizó y cuenta con pacientes y equipo propio en su despacho. El quinto es hijo de un licenciado y su madre cuenta con carrera técnica, trabaja en el consultorio con su padre, no se ha independizado. Y la última es hija de un agrónomo y una odontóloga, desde que terminó sus estudios laboró en dos consultorios privados durante dos años, ya cuenta con su propio espacio mismo que cerró durante un año que estuvo de intercambio académico especializándose en Estados Unidos y ya lo abrió nuevamente. Este conjunto de egresados se explica su totalmente con lo formulado en la

\footnotetext{
${ }^{7}$ Cabe mencionar que han surgido voces críticas al interior de esta corriente del pensamiento económico donde manifiestan que la diferenciación de los pagos por trabajo calificado que se obtiene en las universidades, tiene cada vez menor influencia porque el avance de los nuevos conocimientos. En lugar de considerarse como una ventaja, es ahora una obligación el mantenerse actualizado en el desarrollo tecnológico (Ospina Ramírez, 2015).

${ }^{8}$ En un estudio realizado a la Universidad de Guadalajara que es la institución a la que corresponde el Centro Universitario de Los Altos, Ávila (2013) llega a afirmar que existen diferencias importantes en cuanto al espectro del origen social de los estudiantes entre los que se ubican en un centro metropolitano y uno regional. La Universidad de Guadalajara cuenta con 6 metropolitanos y 8 regionales; los primeros están asentados en la zona metropolitana de Guadalajara y el resto en el interior del estado de Jalisco.
} 
Teoría del Capital Cultural a excepción de que no tuvieron éxito escolar. El Capital Cultural, de acuerdo al máximo exponente de la teoría, se va incorporando a la persona hasta que forma parte de él, y desaparece cuando la persona muere (Bourdieu; 1987)

\section{Conclusiones}

En la primera parte, la que corresponde a la aplicación de una encuesta a los estudiantes de reciente ingreso, destaca conocer que una proporción muy cercana a la mitad de los estudiantes provienen de poblaciones que no pertenecen al área de influencia del Centro Universitario de Los Altos, es el caso de los alumnos de odontología y del resto de las áreas de la salud, no así de las carreras "tradicionales" como administración, contaduría y abogado, o de las ingenierías agroindustrial y en sistemas pecuarios. La explicación la encontramos en que para ingresar a odontología o medicina se requiere un mayor puntaje en las calificaciones obtenidas en la preparatoria y en que hay mayor competencia en esas carreras que se ofrecen en la zona metropolitana de Guadalajara. No obstante la información anterior, es halagador el saber que la proporción de alumnos provenientes de otras zonas ha venido en disminución puesto que al entrevistar a los egresados se pudo conocer que la proporción era mayor tres años antes (de 56.25 a 43\%). Los motivos por los cuales los estudiantes de odontología eligieron esa opción profesional son muy diferentes a los que tienen quienes optaron por otras carreras, los primeros, explican motivos de los principales como el "servir a los demás" mientras que de otras, la razón de que "es la que estaba más cerca de mi domicilio" es de mayor contundencia. Otro escenario diferenciador lo constituye el hecho de pretender emplearse en un espacio propio cuando en la gran mayoría de las opciones universitarias lo que interesa es ubicarse en el ámbito público o privado, muy pocos proyectan crear su propia plaza.

En la segunda parte se pudo dar cuenta de las características laborales de los egresados, exponiendo influencias particulares tales como el origen del financiamiento de su formación, que en el caso de los alumnos de odontología es considerablemente diferente a los de otras carreras: en concreto, la proporción de los estudiantes que se sostienen económicamente por sí mismos es ostensiblemente mayor en otras carreras que en odontología, es decir, esta carrera "es cara". El promedio de escolaridad de los padres de familia es sensiblemente mayor de los estudiantes de odontología que del resto de opciones 
profesionales. Este aspecto es fundamental para determinar el grado de acumulación de capital cultural que se tiene en el seno familiar porque es base de una de las principales explicaciones teóricas del éxito o fracaso laboral. Con respecto al espacio laboral en el cual se desempeñan los padres de los egresados, la característica principal reside en que hay dos grupos muy diferenciados: en uno de ellos la totalidad de los padres son profesionistas y en particular con la formación de odontólogos; en el otro, todos se ubican en espacios laborales de los estratos sociales bajos o a lo más, medios; se dedican a las más variadas formas de oficios (mecánicos, electricistas, choferes, soldadores) que no tienen ninguna posibilidad de acumular capital cultural. Con respecto ya al trabajo al que han podido o no acceder los egresados, presenta dos características también muy marcadas: por un lado, una proporción considerablemente alta consiguió el sueño de las expectativas de oro, es decir, logró establecer su propio consultorio. El 30\% de los estudiantes de primer ingreso se había planteado esa meta y la proporción de los egresados alcanzó un nada despreciable 17\%. No obstante, hablando de la segunda peculiaridad, un $46.80 \%$ se encuentra desempleado y esa cantidad los coloca como la proporción más alta del total de egresados del Centro Universitario de Los Altos. Una cualidad importante a resaltar y que en pocos estudios de este género se toman en cuenta, es el hecho de que el llamado currículum oculto ha dado buenos resultados por la cantidad de conocimientos prácticos que afirman los egresados haber obtenido con el apoyo de sus mentores.

En la tercera parte, la experiencia de un empleador que a su vez es funcionario y profesor, nos ofrece muchas luces para entender las limitaciones y entender las singularidades del empleo de los odontólogos. La primera razón es la cuestión financiera que representa para muchos la decisión más fuerte de si solicitar o no el ingreso al nivel superior de la educación en el área de la odontología. Las mismas razones económicas son el principal obstáculo para la sobrevivencia en la universidad, y también, el factor dinero es fundamental para acceder a la práctica laboral. Lo singular del empleo de los odontólogos es la razón financiera, esta cualidad más que otras es la que determina el éxito o el fracaso en el ingreso, el transcurso y la ocupación una vez egresados. Las tradiciones familiares tienen una influencia definitoria para muchos profesionistas de la odontología, ninguna otra carrera tiene tan acentuada esta característica. 
La última parte, en referencia a la utilidad de los recursos teóricos de las dos corrientes opuestas, en base a los resultados obtenidos como producto de la parte de la investigación cualitativa, se pudo constatar que en el caso de los estudiantes que obtuvieron los más altos logros académicos hablando de las evaluaciones, en la gran mayoría no coincide con que los estudiantes hayan sido hijos de profesionistas y que los trabajos de sus mentores fueran de los más cotizados socialmente. Sin embargo, al considerar al grupo de quienes se posicionaron en un buen espacio laboral, sí existe alta relación con haber sido los hijos de los profesionistas quienes mejor se cotizaron. La Teoría del Capital Humano ofreció luces en cuanto a que los egresados que encontraron un espacio en el mercado de trabajo, ascendieron en la escala social por sus propios méritos, no porque las condiciones familiares les fueran favorables.

En resumen, lo singular del empleo de los profesionistas del área de la odontología, consiste en su resultado dual: que la proporción más alta de sus egresados con respecto a los de otras carreras, no encuentra empleo aun y cuando el espectro social del que provienen es sustancialmente más alto en cuanto al nivel escolar de sus padres. Y segundo, que una minoría que proviene de contextos familiares favorables, encuentra espacio laboral en las mejores condiciones del mercado, es decir, tiene la posibilidad de crear un consultorio propio. 


\section{Bibliografía}

Ávila Enciso, M. (2013). El origen social de los graduados y la equidad en el acceso a la universidad. Revista de la educación superior, 11-29.

Bourdieu, P. (1987). Los tres estados del capital cultural. Sociológica, 11-17.

Bourdieu, P. (1997). Capital cultural, escuela y espacio social. México.: Siglo XXI.

Bourdieu, P. (2002). Premisas y conceptos básicos.

Casillas, M., Chain, R., \& Jácome, N. (2007). Origen social de los estudiantes y trayectorias estudiantiles en la Universidad Veracruzana. Revista de la educación superior, 7-29.

Formichella, M. M., \& London, S. (2013). Empleabilidad, educación y equidad social. Revista de estudios sociales 47, 79-91.

González Pérez, C., \& Carrillo Torres, P. A. (2015). Seguimiento de egresados de la Carrera de Ingeniría en Computación. Guadalajara, México.

González Pérez, C., \& Ortega Cervantes, G. (2015). Empleabilidad de los egresados de la Carrera de Contaduría Pública. Revista Iberoamericana para la Investigación y el Desarrollo Educativo, 1-21.

González Pérez, C., \& Padilla Ruezga, J. A. (2016). El ámbito de la formación y el empleo de los egresados de la Carrera de administración del Centro Universitario de Los Altos. Revista Iberoamericana para la Investigación y el Desarrollo Educativo, 120.

Ospina Ramírez, D. E. (2015). Capital humano: una visión desde la teoría crítica. Cadernos EBAPE. BR 13.2, 315-331. 